\title{
Low Writing Field on Perpendicular Nano-ferromagnetic
}

\author{
Nur Aji Wibowo ${ }^{1 *}$, Ferdy S Rondonuwu², and Budi Purnama ${ }^{3}$ \\ ${ }^{1,2}$ Physics Department, Faculty of Science and Mathematics, Satya Wacana Christian University, Salatiga, Indonesia \\ ${ }^{3}$ Physics Department, Faculty of Mathematics and Natural Science, Sebelas Maret University, Surakarta, Indonesia
}

(Received 3 July 2014, Received in final form 10 August 2014, Accepted 3 September 2014)

\begin{abstract}
For heat-assisted magnetic recording, magnetization reversal probabilities of nano-Pt/MnSb multilayer film with perpendicular magnetic anisotropy under thermal pulse activation were investigated numerically by solving the Landau-Lifshift Gilbert Equation. Magnetic parameters of nano-Pt/MnSb multilayer were used with anisotropy energy of $3 \times 10^{5} \mathrm{erg} / \mathrm{cc}$ and saturation magnetization of $2100 \mathrm{G}$, which offer more than $10 y$ data stability at room temperature. Scheme of driven magnetic field and thermal pulse on writing mechanism was designed closely to real experiment. This study found that the chosen material is potential to be used as a high density magnetic storage that requires low writing field less than two-hundreds Oersted through definite heating and cooling interval. The possibility of writing data with a zero driven magnetic field also became an important result. Further study is recommended on the thickness of media and thermal pulse design as the essential parameters of the reversal magnetization.
\end{abstract}

Keywords : HAMR, magnetization, reversal probability, cooling time, writing field

\section{Introduction}

Within the last two decades, the development of magnetic storage among devices that require large storage capacity has been showing an important progress. Areal density and induced field consume become the focus on data storage technology [1]. One of the branded media that promise ultrahigh areal density attainment is storage media with strong perpendicular magnetic anisotropy that can be realized on $<100 \mathrm{~nm}$ ultra-thin film $[2,3]$. This kind of material enables the creation of small-sized storage devices with high thermal stability, reaching more than $60 k_{\mathrm{B}} \mathrm{T}$ of ambient thermal stability factor, thus ensuring stable data storage for approximately 10 years [4-7]. Nevertheless, this specification requires a highly induced field to overcome high coercivity in data writing process, which is not compatible in technical support. As proposed by Katayama (1999), heat assisted technology could be wielded to solve this problem [8]. Heat-assisted magnetic recording (HAMR) heats the local region of a storage medium before cooling it under the settled induced field in appropriate magnetic polarity $[4,7]$. Since its emergence, HAMR has

(C)The Korean Magnetics Society. All rights reserved.

*Corresponding author: Tel: +62-8-572-5580282

Fax: +62-29-832-1433, e-mail: nurajiwibowo@gmail.com replaced conventional magnetic recordings due to its superior properties that offer continuing expansion on storage capacities with high thermal stability $[9,10]$. Along with HAMR development, thermal issues that become the problem in data writing must be addressed in order to attain high areal density of a magnetic storage device as long as the required writing field magnitude is applicable (it does not exceed $17 \mathrm{kOe}$ ) $[7,9,11,12]$. One aspect to address is cooling time $[4,15]$. Recent studies report that time magnetization precision of about $100 \mathrm{GHz}$ is a realistic mechanism, which value of which is correlated to the cooling time of hundreds of pico-second in HAMR application $[13,14]$.

In this study, laser heating and writing field pulse were simulated to investigate the impact of cooling time on the writing field magnitude through micromagnetic simulation by solving the Landau-Lifshift Gilbert equation, which involves randomized magnetization and magnetic field fluctuation as a consequence of localized heating pulse.

\section{Numerical Method}

Micromagnetic simulation for thermally assisted magnetization reversal evaluation has been performed using the modified micromagnetic simulator created by $\mathrm{H}$. Takamatsu, 
K. Sakai, and S. Konishi from Kyushu University, Japan, by applying thermal aspect. This numerical analysis was based on an explicit Landau-Lifshift Gilbert equation with a thermal fluctuation field as an additional contribution on effective field to accomplish some previous field contributors, such as demagnetization field, exchange field, anisotropy field, and Zeeman field. The mean value of the thermal fluctuation field over the magnetization process was counted as zero value, $\left\langle H_{T}^{i}(t)\right\rangle=0$, and was considered as Gaussian distribution, $\left\langle H_{T}^{i}(t) H_{T}^{j}\left(t^{\prime}\right)\right\rangle=\delta_{i j} \delta\left(t-t^{\prime}\right) \sigma^{2}$, with $\sigma$ assigned by the fluctuation-dissipation theorem $\sigma=\sqrt{2 k_{B} T \alpha / \gamma V M_{s} \Delta t}$, where $k_{\mathrm{B}}=$ Boltzman constant, $T=$ temperature, $\alpha=$ Gilbert damping factor $(0.3), \gamma=$ gyromagnetic ratio $\left(1.76 \times 10^{7} \mathrm{Oe}^{-1} \cdot \mathrm{s}^{-1}\right), V=$ volume of media, $M_{\mathrm{s}}=$ magnetic saturation, and $\Delta t=$ time increment $\left(0.25 \times 10^{-12} \mathrm{~s}\right)$ [16-19].

Storage media used to record one bit information consisted of parallel-pipes, called nano-dots, with lateral dimension of $50 \times 50 \mathrm{~nm}^{2}$ in $y z$ direction and a thickness of $<100 \mathrm{~nm}$ in $x$ direction to design a ultra-thin strong perpendicular magnetic anisotropy media that allows data record up to hundreds of $\mathrm{Gb} / \mathrm{in}^{2}$. The Nano-dot was composed from unit cells with lateral size of $3.3 \times 3.3 \mathrm{~nm}^{2}$ in which each cell represents a single magnetic polarization and magnetized along $x(-)$ direction at initial condition. Nano-dots was considered as an ideal isolated particle, which interaction between neighboring nano-dots in storage media system was neglected.

The magnetic substance chosen to be analyzed in this simulation is $\mathrm{Pt} / \mathrm{MnSb}$ multilayered films. As one of Heusler Alloys, it has larger spin polarization than $\mathrm{CoMnSb}$ [20]. This material characteristic is a key factor in determining spintronic devices performance [21]. It has flexibility in changing magnetic properties through the manufacturing process, such as changing the annealing temperature and period [22]. The magnetic properties used in this simulation are Currie temperature $\left(T_{\mathrm{C}}\right)$ as large as 373 $\mathrm{K}, 3 \times 10^{5} \mathrm{erg} / \mathrm{cc}$ in anisotropy constant $\left(K_{\perp}\right), 2100 \mathrm{G}$ in magnetic saturation $\left(4 \pi M_{\mathrm{s}}\right)$, and $10^{-7} \mathrm{erg} / \mathrm{cm}$ in exchange stiffness constant $(A)$. The last three magnetic property values mentioned above were at ambient temperature. As a consequence of thermal field appearance, their temperature dependence was considered similar with previous paper which has been done by Purnama et al. (2009) for stochastic simulation of thermally assisted devices, as shown in Eq. (1)-(3) [23]. Purnama and colleagues define the criteria of magnetization reversal as $85 \%$ of actual magnetization $(M)$, parallel to driven magnetic field direction $(H)$.

$$
K_{\perp}(T)=K_{\perp}^{(0)}\left(\frac{M_{s}(T)}{M_{s}(0)}\right)^{2}
$$

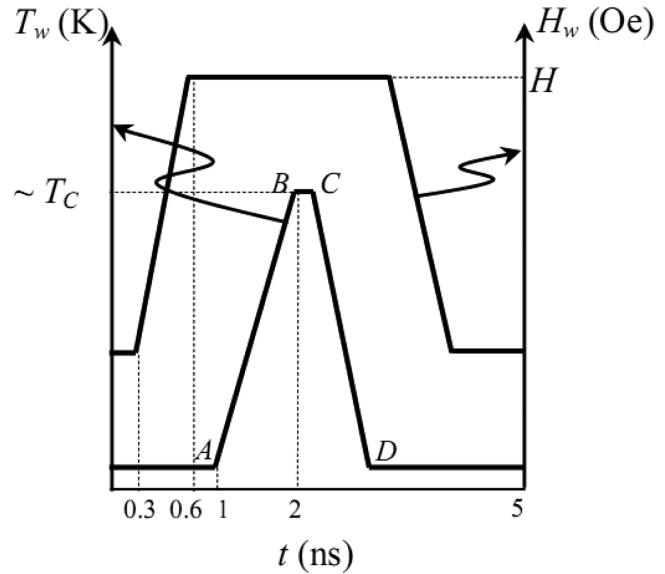

Fig. 1. Graph of the ideal external magnetic field and the thermal pulse on the writing mechanism.

$$
\begin{aligned}
& M_{s}(T)=M_{s}^{(0)}\left(1-T / T_{C}\right)^{0.5} \\
& A(T)=A^{(0)}\left(\frac{M_{s}(T)}{M_{s}(0)}\right)^{2}
\end{aligned}
$$

In order to examine the cooling effect on the writing mechanism, in this study, thermal pulse $\left(T_{\mathrm{w}}\right)$ as well as magnetic field pulse $\left(H_{\mathrm{w}}\right)$ were schemed as described in Figure 1. After the driven magnetic field was achieved, the heating pulse started to increase linearly up to $372.9 \mathrm{~K}$ (A-B), close to its Currie temperature. The linear increasing type for both, external magnetic field and heating pulse, were chosen for ideal models. Then, this pulse constantly heated up the nano-dot for 187.5 pico-seconds (B-C) to randomize its magnetization. Finally, the nanodot temperature dropped (C-D) under the external field treatment to freeze its magnetization polarity. Therefore, the interval during C-D process is called cooling time $\left(t_{\mathrm{c}}\right)$.

\section{Result and Discussion}

To ensure that nano-Pt/MnSb accomplishes the minimum requirement of life time Hard Disk Drive, the barrier energy at room temperature must be calculated. In this study, the chosen parameters have sufficiently large barrier energy for $10 \mathrm{~nm}$ and $20 \mathrm{~nm}$ in thickness, thus promising more than $10 y$ data storage stability. The magnetization reversal of nano-dot under induced magnetic field as well as thermal pulse visualized in micrograph is presented in Fig. 2. $M / M_{\text {sat }}$ represents the normalized value of actual magnetization compared to the initial saturated magnetization $\left(M_{\text {sat }}\right)$. In the micrograph, black color represents the magnetization in $x(-), M \downarrow$ and white color in $x(+), M \uparrow$ (parallel to applied magnetic field direction), and vice versa. Before the first nano-second, $M / M_{\text {sat }}$ is equal to -1 , 


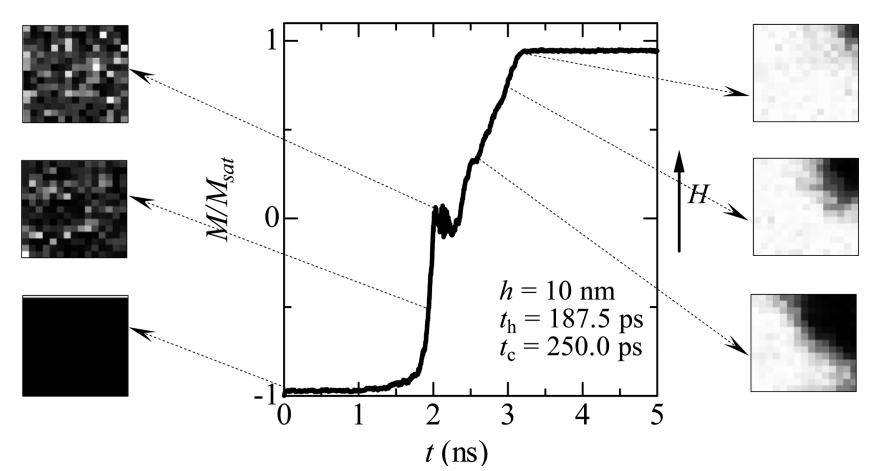

Fig. 2. Magnetization of the nano-dot during the writing mechanism on nano $\mathrm{Pt} / \mathrm{MnSb}$ for $t_{\mathrm{h}}=187.5 \mathrm{ps}, t_{\mathrm{c}}=250.0 \mathrm{ps}$, and $h=10 \mathrm{~nm}$.

which denotes that magnetization occurred even before the magnetic field was induced. However, magnetization started to change when the thermal pulse was activated and varied drastically through the multi-domain configuration mechanism when the nano-dot temperature was close to its Currie point. The random magnetization state was achieved at $2 \mathrm{~ns}$, when $T=372.9 \mathrm{~K}$ for $187.5 \mathrm{ps}$, in which the nano-dot does not have any magnetization tendency even though the external magnetic field is offered. Sub-nanosecond of heating interval was adopted in order to realize fast heating as an assistance to reverse magnetization. After reducing the temperature, the nano-dot magnetization was adjusted parallel to applied magnetic field by means of the domain wall nucleation, which is going to propagate until it coincides with the field direction at room temperature, by means of single domain configuration that was achieved with the corresponding value of $M / M_{\text {sat }}$ being equal to 0.85 .

The thermal fluctuation effect during the magnetization

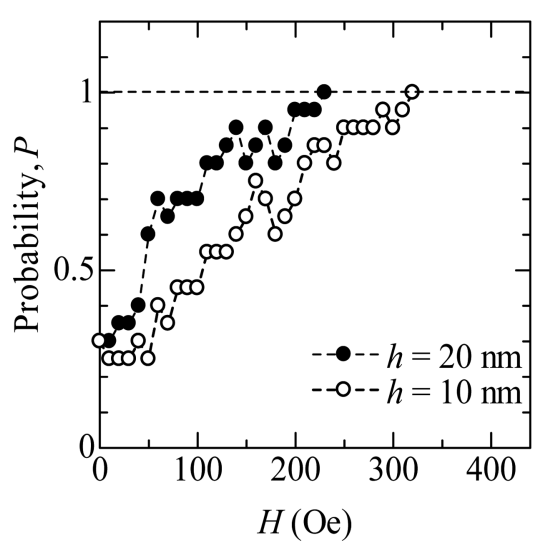

(a) reversal process was evaluated by adopting 20 random numbers of disordered magnetization. The typical increase of reversal probabilities over the given random number actually depends on the induced magnetic field magnitude as shown in Fig. 3(a). As the magnetic field increased from zero, reversal probability raised. At a specific magnetic field value, which is called threshold field $\left(H_{\text {th }}\right)$, this probability starts to reach 1 and keep this value continuously for larger $H$. For $H>H_{\mathrm{th}}$, the reversal probabilities are constantly equal to one, which means that the nanodot magnetization assuredly lies on the external magnetic field direction. Ostler et al. posited that ultrafast heating can reverse magnetization even on a zero driven magnetic field [24]. The phenomena of reversal magnetization under thermally stimulation without magnetic field induction is also observed in this study as shown in Fig. 3(b). On the heating of hundreds of pico-seconds, the reversal magnetization probability values were more than zero for numerous given cooling times even when the external magnetic field was not applied. The nano-dot with a thickness of 20

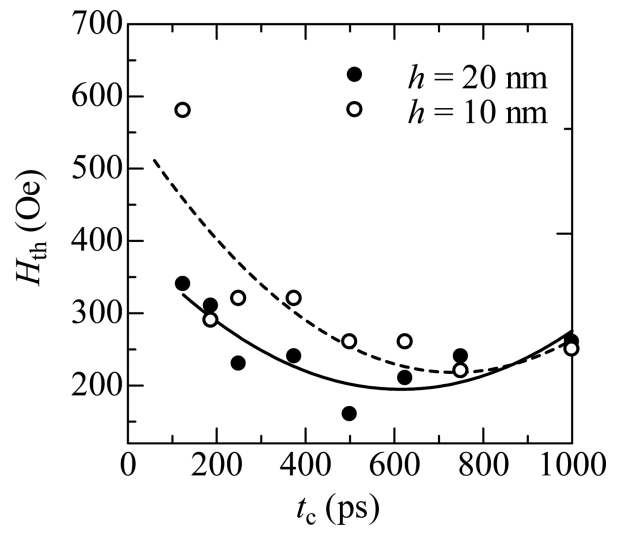

Fig. 4. Threshold field differs from the cooling interval.

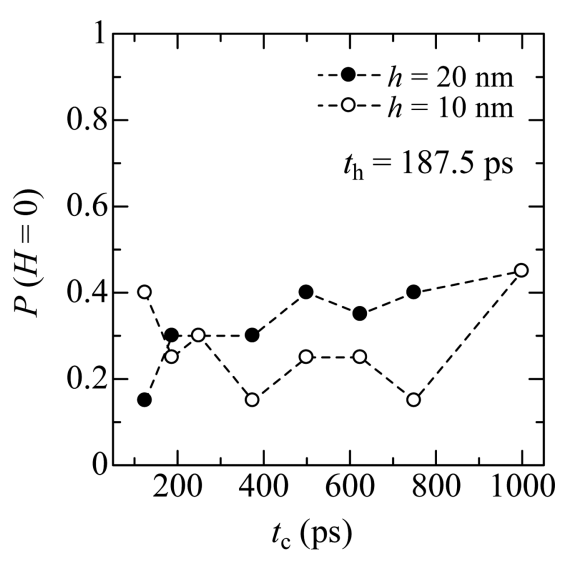

(b)

Fig. 3. (a) Reversal probabilities of nano-dot over twenty given random number for $t_{\mathrm{c}}=250.0 \mathrm{ps}$, (b) reversal probabilities when no field is presented. 
$\mathrm{nm}$ has a more significant increase to $P(H=0)$ with respect to cooling interval than another one and the demagnetization energy becomes an important factor that must be considered.

A longer cooling process also influences the threshold field level. As it is illustrated in Fig. 4, a short cooling phase generally increased the threshold field. Within the given cooling interval, the thicker nano-dot corresponded to lower threshold field and had a minimum value of about $160 \mathrm{Oe}$, a much smaller writing field compared to the writing heads available on the market. Both profiles indicate that there is an optimum cooling time to obtain the lowest writing field for each thickness.

\section{Conclusion}

The effect of cooling time on the magnitude of the threshold field, which in turn influences the writing field on a perpendicular nano-Pt/MnSb multilayer, was numerically examined. From the adopted nano-dot model with its magnetic properties, the micromagnetic study shows that a high density of recording media up to hundreds $\mathrm{Gb} /$ $i^{2}$ can be realized in a low writing field $(<200$ Oe) through definite heating and cooling period. It generates optimism about minimizing the power consumption on high recording density read-write data technology. This study also found that the reversal magnetization is possible to realize, but only with an ultrafast heating mechanism. A further study is needed to examine the contribution of the thickness of media and the cooling interval regarding the reversal magnetization probabilities.

\section{References}

[1] N. A. Wibowo, and B. Purnama, IACSIT Int. J. Eng. Technol. 3 (2011). Doi: 10.7763/IJET.2011.V3.256.

[2] S. H. Lim, and H. J Kim, J. Magn. 6, 109 (2001).

[3] Sabine Alebrand, Matthias Gottwald, Michel Hehn, Daniel Steil, Mirko Cinchetti, Daniel Lacour, Eric E. Fullerton, Martin Aeschlimann, and Stephane Mangin, Appl. Phys. Lett. 101, 162408 (2012).

[4] Mark H. Kryder, Edward C. Gage, Terry W. McDaniel, William A. Challener, Robert E. Rottmayer, Ganping Ju, Yiao-Tee Hsia, and M. Fatih Erden, Proceedings of the Institute of Electrical and Electronics Engineers (invited paper) 96, 11 (2008).

[5] S. H. Yoon, and K. M. Krishnan, J. Appl. Phys. 109, 07B534 (2011).

[6] Ralph Skomski, J. Appl. Phys. 101, 09B104 (2007).

[7] Koji Matsumoto, Akhihiro Inomata, and Shin-ya Hasigawa, Fujitsu Scientific \& Technical Journal 42, 158 (2006).
[8] Hiroyuki Katayama, Shinzo Sawamura, Yasushi Ogimoto, Junsaku Nakajima, Kunio Kojima, and Kenji Ohta, J. Magn. Soc. Japan 23, 233 (1999).

[9] Sari Shafidah Binte Shafiee, Moulay Rachid Elidrissi, Hong Tao Wang, Kwaku Eason, Rathna Kumar Radhakrishnan, Kheong Sann Chan, and Yong Liang Guan, J. Appl. Phys. 111, 07B714 (2012).

[10] R. H. Victora, IEEE Trans. Magn. 49, 2 (2013).

[11] G Vinai, J Moritz, S Bandiera, I L Prejbeanu, and B Dieny, J. Phys. D: Appl. Phys. 46, 322001 (2013). Doi:10.1088/0022-3727/46/32/322001.

[12] B. X. Xu, Z. J. Liu, R. Ji, Y. T. Toh, J. F. Hu, J. M. Li, J. Zhang, K. D. Ye, and C. W. Chia, J. Appl. Phys. 111, 07B701 (2012).

[13] U. Kilic, G. Finocchio, T. Hauet, S. H. Florez, G. Aktas, and O. Ozatay, Appl. Phys. Lett. 101, 252407 (2012).

[14] O. Ozatay, T. Hauet, S. H. Florez, J. A. Katine, A. Moser, J.-U. Thiele, L. Folks, and B. D. Terris, Appl. Phys. Lett. 95, 172502 (2009).

[15] Keita Waseda, Ryosuke Doi, Budi Purnama, Satoru Yoshimura, Yukio Nozaki, and Kimihide Matsuyama, IEEE Trans. Magn. 44, 2483 (2008).

[16] K. J. Lee and T. D. Lee, J. Appl. Phys. 91, 7706 (2002).

[17] Budi Purnama, Thermally Assisted Magnetization Reversal in Perpendicularly Magnetized Thin Film, (Doctoral Thesis), Electronics Department Graduated School of Information Science and Electrical Engineering, Kyushu University, Japan (2009), pp. 12-43.

[18] T. Schrelf, J. Fidler, D. Suess, W. Scholz, and V. Tsiantos, Handbook of Advanced Magnetic Materials: Micromagnetic Simulation of Dynamic and Thermal Effects. Volume I, Chanpter 4, Tsinghua University Press, China (2006) pp. 128-146.

[19] D. P. Agustina Candra, Suryasatriya Trihandaru, and Nur Aji Wibowo, Int. J. Sci. Res. 2, 48 (2013).

[20] I. Galanakis, J. Phys.: Conden. Matt. 14, (2002). Doi: 10.1088/0953-8984/14/25/303.

[21] Koichiro Inomata, Naomichi Ikeda, Nobuki Tezuka, Ryogo Goto, Satoshi Sugimoto, Marek Wojcik, and Eva Jedryka, Science and Technology of Advanced Materials 9, (2008). Doi: 10.1088/1468-6996/9/1/014101.

[22] T. Kawanabe, and M. Naoe, Journal de Physique, Colloque C8, Supplement au no 12 , Tome 49, C8-1783-1784 (1988). Available at http://dx.doi.org/10.1051/jphyscol: 19888813.

[23] Budi Purnama, Masashi Koga, Yukio Nozaki, and Kimihide Matsuyama, J. Magn. Magn. Mater. 321, 1325 (2009).

[24] T. A. Ostler, J. Barker, R. F. L. Evans, R. W. Chantrell, U. Atxitia, O. Chubykalo-Fesenko, S. El Moussaoui, L. Le Guyader, E. Mengotti, L. J. Heyderman, F. Nolting, A. Tsukamoto, A. Itoh, D. Afanasiev, B. A. Ivanov, A. M. Kalashnikova, K. Vahaplar, J. Mentink, A. Kirilyuk, Th. Rasing, and A. V. Kimel, Nature Communications 3, 666 (2012). Doi: 10.1038/ncomms1666. 